\title{
Interaction of cylindrical polymer brushes in dilute and semi-dilute solution
}

\author{
Sreenath Bolisetty $\cdot$ Sabine Rosenfeldt . \\ Christophe N. Rochette • Ludger Harnau • \\ Peter Lindner • Youyong Xu • Axel H. E. Müller • \\ Matthias Ballauff
}

Received: 3 September 2008 /Revised: 24 October 2008 / Accepted: 29 October 2008 / Published online: 6 December 2008

(C) Springer-Verlag 2008

\begin{abstract}
We present a systematic study of flexible cylindrical brush-shaped macromolecules in a good solvent by small-angle neutron scattering (SANS), static light scattering (SLS), and by dynamic light scattering (DLS) in dilute and semi-dilute solution. The SLS and SANS data extrapolated to infinite dilution lead to the shape of the polymer that can be modeled in terms of a worm-like chain with a contour length of $380 \mathrm{~nm}$ and a persistence length of $17.5 \mathrm{~nm}$. SANS data taken at higher polymer concentration were evaluated by using the polymer reference interaction site model (PRISM). We find that the persistence length reduce from $17.5 \mathrm{~nm}$ at infinite dilution to $5.3 \mathrm{~nm}$ at the highest concentration (volume fraction 0.038). This is comparable with the decrease of the persistence length in
\end{abstract}

S. Bolisetty $\cdot$ S. Rosenfeldt $(\triangle) \cdot$ C. N. Rochette $\cdot$ M. Ballauff Physikalische Chemie I, University of Bayreuth, 95440 Bayreuth, Germany

e-mail: Sabine.Rosenfeldt@uni-bayreuth.de

L. Harnau

Max-Planck-Institut für Metallforschung,

Heisenbergstrasse 3,

70569 Stuttgart, Germany

e-mail: harnau@fluids.mpi-stuttgart.mpg.de

L. Harnau

Institut für Theoretische und Angewandte Physik,

Universität Stuttgart,

Pfaffenwaldring 57,

70569 Stuttgart, Germany

P. Lindner

Institut Laue-Langevin,

Grenoble Cedex, France

Y. Xu • A. H. E. Müller

Makromolekulare Chemie II, University of Bayreuth,

95440 Bayreuth, Germany semi-dilute concentration predicted theoretically for polyelectrolytes. This finding reveals a softening of stiffness of the polymer brushes caused by their mutual interaction.

Keywords Cylindrical polymer brushes .

Softening of polymers $\cdot$ SANS $\cdot$ DLS $\cdot$ PRISM

\section{Introduction}

If polymeric side chains are grafted to a polymer backbone, a cylindrical polymer brush results [1-3]. In recent years, these polymers have become the subject of intense experimental and theoretical research interest by a broad variety of methods $[4,5]$. The main feature of cylindrical brushes is a marked stiffening of the main chains. It has been shown theoretically and by computer simulations [6] that this stiffening is due to a balance of repulsive forces originating from a steric overcrowding of the side chains and the entropic restoring forces of the main chain. The analysis of cylindrical brushes by small-angle neutron scattering (SANS) [7, 8], small-angle X-ray scattering (SAXS) [9] and static light scattering (SLS) [10] in dilute solution has demonstrated that these macromolecules exhibit a worm-like conformation that may be approximated by a cylinder if the length of the brush is not too large. This is in qualitative agreement with studies of the contour length and end to end distance of individual brush molecules in the dried state by atomic force microscopy (AFM) [11]. Up to now, most studies on cylindrical brushes in solution have been conducted in the dilute regime. Recently, Rathgeber et al. [10] reported the formation of ordered structures of dissolved brushes at intermediate concentrations. However, still higher concentrations lead to the disappearance of the ordered phase and thus to a 
reentrant isotropic phase. The authors explained this by a screening of the excluded volume interaction when the polymers start to overlap. However, no quantitative explanation was given.

The central question of this investigation is the interplay between the interaction and the stiffness of the brushes in dilute and semi-dilute solution. Following the decrease of the persistence length by mutual interaction, we aim at a general discussion of concentration-dependent conformational changes of chain molecules. The decrease of the persistence length by mutual interaction has first been discussed for cylindrical brushes by Borisov et al. in 1987 [12]. For polyelectrolytes in solution, Stevens and Kremer demonstrated that an increase of the concentration must lead to a decrease of the persistence length [13]. Cylindrical polymer brushes offer the possibility to study the softening of the stiffness in a neutral system. Scattering methods allows in situ studies and therefore give access to this important problem related to polymer solutions. A quantitative understanding of the correlations and interaction of polymeric species can be achieved using the polymer reference interaction site model (PRISM) integral equation theory. Here we use the PRISM theory to model the interaction of cylinder brushes at finite concentrations.

Recently, we investigated a worm-like polymer brush near the overlap concentration and detected a softening of the bottle-brush at high concentrations due to mutual interaction with the other brushes [14]. Here we give a full account of this work.

Figure 1 displays the chemical structure of the polymer studied here. The brush was synthesized by a "grafting from" method and composed of poly(2-hydroxyethylmethacrylate) (P-HEMA) backbone grafted with poly(t-butyl acrylate)(P-

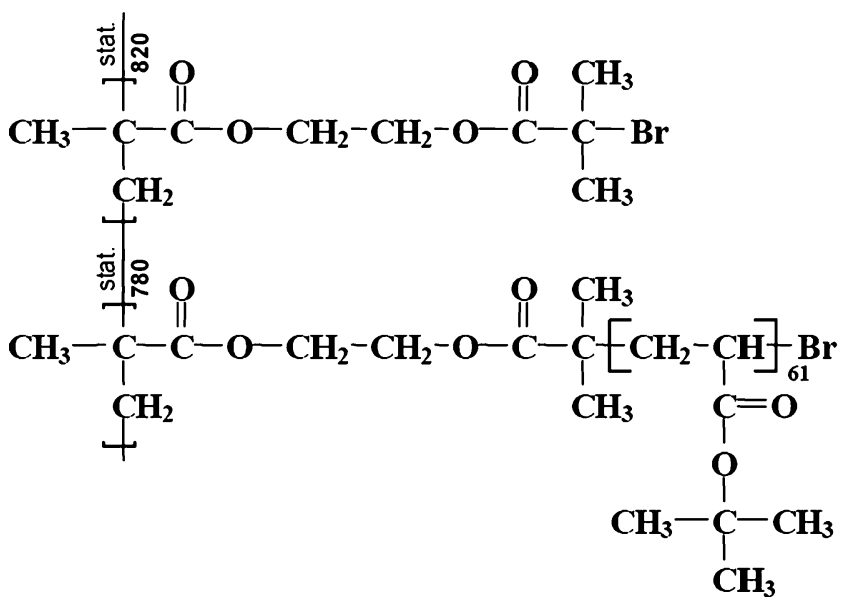

Fig. 1 Chemical structure of the investigated cylindrical polymer brush consisting of a poly(2-hydroxy-ethylmethacrylate) (P-HEMA) backbone grafted with poly(t-butyl acrylate)(P-TBA) chains. The repeating units carrying side chains (ca. 780) alternate statistically with unsubstituted repeating units (ca. 820). The weight average number of repeating units per side chain is 61
TBA) chains. The weight average number of repeating units in the main chain is 1,600 with a polydispersity index of $M_{\mathrm{W}} / M_{\mathrm{n}}=1.06$. Due to the initiator efficiency of approximately 0.5 only every second repeating unit carries a side chain containing ca. 61 units. Thus, the brush is a statistical copolymer of units with side chains (ca. 780) and units without side chains (ca. 820). Hence, we deal with a rather sparse brush system as opposed to dense brushes in which each repeating unit carries a side chain. The details of the synthesis have been reported in reference [4].

The article is organized as follows: first we present a characterization of the brush macromolecules in dilute solution in order to characterize its molecular structure and bare persistence length measured at infinite dilution. Next, more concentrated solutions will be analyzed. The interaction between the brushes will be modeled using the PRISM integral equation theory. In the third section the dynamic properties of the polymer brushes will be considered.

\section{Experiment}

\section{Small-angle neutron scattering}

The poly(t-butyl acrylate) brush was synthesized by "grafting from" route via atom transfer radical polymerization leading to a (poly 2-hydroxyethylmethacrylate) backbone partially grafted with poly(t-butylacrylate) (P-TBA) side chains. Details have been reported before [3-5, 14]. Deuterated tetrahydrofuran $\left(\right.$ THF- $\mathrm{d}_{8}$, Fluka, deuteration degree $99.9 \%$ ) was used as received. The partial specific volume of the polymer was determined using a DMA-60densitometer (Paar, Graz, Austria) to $\bar{v}=1.10( \pm 0.02) \mathrm{cm}^{3} / \mathrm{g}$. All SANS data were obtained using the instrument D11 of the Institute Laue-Langevin (ILL) in Grenoble (France). The wavelength of the incident neutrons was $0.6 \mathrm{~nm}$ and the sample-detector distances were set to $1.1,5$, and $20 \mathrm{~m}$. In order to obtain the radial averaged intensities in absolute scale, the data were treated by the use of the software provided at the instrument. For all data sets, the rates of incoherent scattering caused mainly by the protons were determined at high scattering angle, set as a constant and subtracted from the crude data. Further treatment was done according to references $[15,16]$.

Light scattering

The polymer brush was dissolved in THF (Sigma Aldrich) leading to a concentration range of $1.5 \mathrm{~g} / \mathrm{L}$ to $7.0 \mathrm{~g} / \mathrm{L}$. The solutions are filtered into dust-free optical cells using $0.45 \mu \mathrm{m}$ PTFE filters. The light scattering experiments were carried out by using the ALV/DLS/SLS-5000 compact goniometer system equipped with a $\mathrm{He}-\mathrm{Ne}$ laser $(\lambda=$ 
$632.8 \mathrm{~nm}$ ). For each solution simultaneous dynamic light scattering (DLS) and static light-scattering (SLS) measurements were performed (three runs of $600 \mathrm{~s}$, angles changing from $30^{\circ}$ to $150^{\circ}$ with an angular step width of $10^{\circ}$ ). The refractive increment $\mathrm{d} n / \mathrm{d} c$ of the solution was measured using a DnDc2010 device supplied by Polymer Standards Service to $0.063 \pm 0.002 \mathrm{ml} / \mathrm{g}$. For depolarized dynamic light scattering (DDLS) measurements a Glan-Thomson polarizer with an extension coefficient better than $10^{-5}$ was used. The light scattering data were analyzed according to the CONTIN method using standard ALV software.

\section{Theoretical basis}

Small-angle neutron scattering and light scattering determine the intensity $I(q, \phi)$ as a function of the scattering vector $q$ and the volume fraction $\phi$ of the dissolved objects. The absolute value of the scattering vector is given by $q=$ $|q|=(4 \pi n / \lambda) \sin (\theta / 2)$ in which $n$ is the refractive index of the medium, $\lambda$ is the incident wavelength, and $\theta$ is the scattering angle. For the systems under consideration the scattering intensity may be rendered as [17]

$I(q, \phi)=\phi(\Delta \rho)^{2} V_{\mathrm{p}} P(q) S(q, \phi)+\phi I_{\text {fluc }}(q)$.

The form factor $P(q)$ is determined by interference effects between radiation scattered by different parts of the same particle. Consequently, this scattering intensity describes the shape of the particle with volume $V_{\mathrm{p}}$. The degree of local order in the sample is given by the structure factor $S(q, \phi)$ which is related to the mutual interactions between different particles. $\Delta \rho$ denotes the excess scattering length density between the solute and the solvent. Finally, the contribution to the scattering intensity due to density fluctuations of the polymer chains is denoted by $I_{\text {fluc }}(q)$. This contribution becomes only important for high scattering vectors.

For dilute solutions, the dependence of the structure factor $S(q, \phi)$ on the volume fraction $\phi\left(\phi=c / \rho_{T}, \rho_{T}\right.$ is the density of the particle and $c$ is the weight concentration of the dissolved polymer) may be expressed in a virial series by $[15,17-20]$

$\frac{1}{S(q, \phi)}=1+2 B_{\text {app }}(q) \phi+O\left(\phi^{2}\right)$.

The apparent second virial coefficient $B_{\text {app }}(q)$ includes the effective diameter of interaction $d_{\text {eff }}$ and the particle volume $V_{\mathrm{p}}$. The apparent second virial coefficient is an explicit function of $q[15,17-19]$

$$
B_{\mathrm{app}}(q)=\frac{2 \pi d_{\mathrm{eff}}^{3}}{3 V_{\mathrm{p}}}\left(1-\frac{1}{10} d_{\mathrm{eff}}^{2} q^{2}+O\left(q^{3}\right)\right) .
$$

In the limit of vanishing scattering vector $B_{\text {app }}(q)$ reduces to its thermodynamic limit, the second virial coefficient. Thus, the effective diameter $d_{\text {eff }}$ gives the balance of both repulsive and attractive interactions between the solute molecules $[15,19]$.

With complete generality, $I(q, \phi)$ may be approximated in the region of small $q$ by Guinier's law [17]

$I(q, \phi) \stackrel{q R_{\mathrm{g}}<<1, \phi \rightarrow 0}{\longrightarrow} \phi V_{\mathrm{p}}(\Delta \rho)^{2} \exp \left(-\frac{R_{\mathrm{g}}^{2}}{3} q^{2}\right)$

where $R_{\mathrm{g}}$ is the radius of gyration. $R_{\mathrm{g}}$ depends on the excess scattering length density $\Delta \rho$.

The scattering intensity of randomly orientated, noninteracting cylindrical objects can be approximated by the product of an infinitely thin rod and a factor which is due to the finite diameter of the chain [17]. At sufficiently high scattering angles this approximation is given by [17]

$I(q, \phi)-\phi I_{\text {fluc }}(q) \stackrel{q R_{\mathrm{g}}>>1}{\longrightarrow} \pi(\Delta \rho)^{2} \phi \frac{V_{\mathrm{p}}}{q L} \exp \left(-\frac{1}{2} R_{\mathrm{c}}^{2} q^{2}\right)$

Here the radius of gyration perpendicular to the chain axis is denoted as $R_{\mathrm{c}}$. From the particle volume $V_{\mathrm{p}}$ the molecular weight $M_{\mathrm{W}}$ can be obtained by $V_{\mathrm{p}}=M_{\mathrm{W}} / N_{\mathrm{A}} / \rho_{T}$ ( $N_{\mathrm{A}}$ is Avogadro's number). The division of the molecular weight $M_{\mathrm{W}}$ by the total length $L$ leads to the mass per unit length $M_{\mathrm{L}}$.

Thus, the forgoing consideration suggest the following way to determine the overall structure of the isolated polymer brush: At first, the data measured at finite concentration are extrapolated to vanishing concentration by use of Eqs. 2 and 3. After subtraction of the scattering contribution due to thermal fluctuations of the side chains the scattering intensity of a single particle $P(q)$ results and the worm-like shape of the investigated polymer brushes can be confirmed. Finally, the structure factor $S(q, \phi)$ obtained according to Eq. 1 can be compared to calculations done within the frame of the PRISM approach.

\section{Results and discussion}

Analysis of the particles in dilute solution

The present analysis rests on precise measurements of $I(q, \phi)$ at different concentrations. The set of data obtained for different concentrations are displayed in Fig. 2 together with the result of the extrapolation to vanishing concentration which includes SANS and SLS data. 


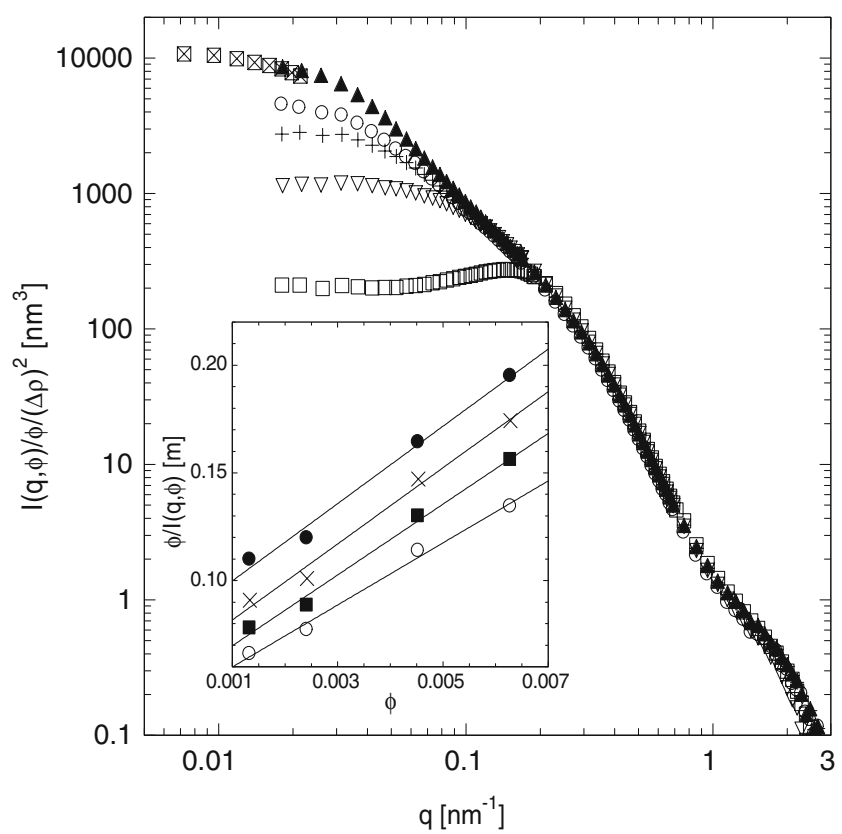

Fig. 2 Influence of concentration on the absolute scattering intensities $I(q, \phi) / \phi . I(q, \phi) / \phi$ as function of $q$. Parameter of the different SANS curves is the volume fraction (top to bottom: $\phi=0.000,0.002,0.006$, 0.013 , and 0.038 ). The extrapolation to $\phi=0.000$ includes the SLS data (filled squares). Inset Determination of $S(q, \phi)$ from the dilute regime by extrapolation for different $q$ values (top to bottom: $q=0.027 \mathrm{~nm}^{-1}$, $0.021 \mathrm{~nm}^{-1}, 0.016 \mathrm{~nm}^{-1}$, and $0.010 \mathrm{~nm}^{-1}$ ). Here SLS data are shown

For the sake of clarity not all data taken at low concentration are shown in the graph. As expected, all measured SANS intensities as the function of concentration differ strongly at low $q$ but superimpose at intermediate and high $q$ values. The suppression of the intensity near $q=0$ (correlation hole effect) affects more than one order of magnitude. This point directly to the effect of mutual interaction of the polymer brushes. Evidently, non-negligible interaction starts at much lower volume concentrations than measured, e.g. below $\phi=0.001$. The extrapolation to vanishing concentration is done separately for each $q$ value as suggested by Eq. 2 (see Fig. 2 inset). Due to statistical problems and low concentrations a linear extrapolation is used. Since we aim at the limits of vanishing $q$ values, the $q$ range accessible by SANS was not sufficient. Hence, static light scattering was used to obtain data at smallest $q$ values possible in order to supplement the analysis by SANS.

Having extrapolated $I(q, \phi) / \phi$ to $\phi=0$, Eq. 3 may be used to determine the effective diameter $d_{\text {eff }}$ of interaction. Figure 3 shows the apparent virial coefficient $B_{\text {app }}(q)$ in the dilute regime as obtained by SLS and SANS as function of $q^{2}$. The data agree within the given limits of error. Figure 3 clearly demonstrates that $B_{\text {app }}(q)$ strongly depends on the scattering angle as expected. The effective diameter $d_{\text {eff }}$ can be calculated from the slope of the linear regression to $d_{\mathrm{eff}}=64 \pm 10 \mathrm{~nm}$.

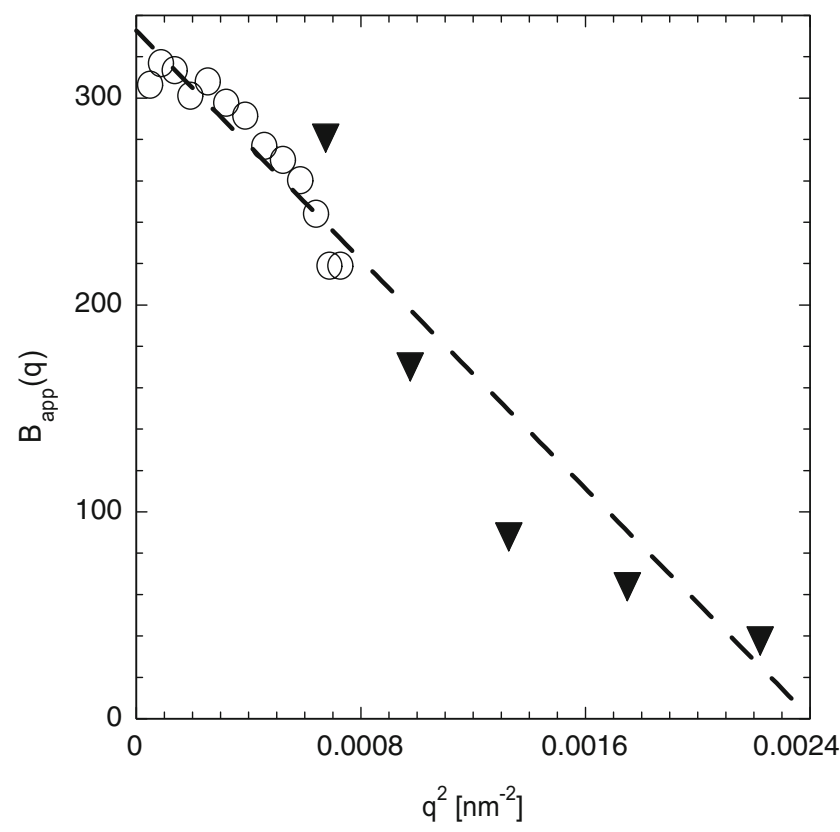

Fig. 3 Apparent second viral coefficient $B_{\text {app }}(q)$ of the polymer brush in the dilute regime as determined by SLS (circles) and SANS (inverted triangles). The effective diameter $d_{\mathrm{eff}}$ deriving from the linear regression results as ca. $64 \mathrm{~nm}$

Figure 4 displays the intensity, extrapolated to vanishing concentration for the entire $q$ range available by the combination of SLS and SANS. In general, the side chains of the polymer brushes exhibit density fluctuations that will give an additional scattering contribution at large $q$ values (cf. Eq. 1). The rather flat region at small $q$ in Fig. 3 is due to the fact that the intensity must follow Guinier's law (Eq. 4) in this regime. The radius of gyration resulting from Guinier's law follows as $R_{\mathrm{g}}=53 \mathrm{~nm}$ and the molecular weight $M_{\mathrm{W}}$ is found to be $7.41 \times 10^{6} \mathrm{~g} / \mathrm{mol}$. Together with the weight-average degree of polymerization $P_{\mathrm{w}}=1,600$ of the main chain this leads to an average molecular weight of the repeating unit of $M_{0}=4,600 \mathrm{~g} / \mathrm{mol}$. Note that this is the average of all repeating units of which only ca. 50\% carry side chains (cf. Fig. 1).

Further, Fig. 4 demonstrates that the scattering intensity is dominated by an additional contribution at $q>0.8 \mathrm{~nm}^{-1}$. The contribution due to internal density fluctuations may be approximated by [7]

$I_{\text {fluc }}(q)=a_{b} \frac{\sin \left[\mu \tan ^{-1}\left(q_{b}{ }^{*}\right)\right]}{\mu q_{b} *\left[1+q_{b}^{*}\right]^{\mu / 2}}$

with

$q_{b}^{*}=\frac{q \xi}{\left[\operatorname{erf}\left(q R_{c} / \sqrt{6}\right)\right]^{3}}$ and $\mu=\frac{1}{v_{b}}-1$

where $v_{b}$ is the Flory exponent, $a$ measure of the fractal dimension, erf denotes the error function. The amplitude $a_{b}$ 


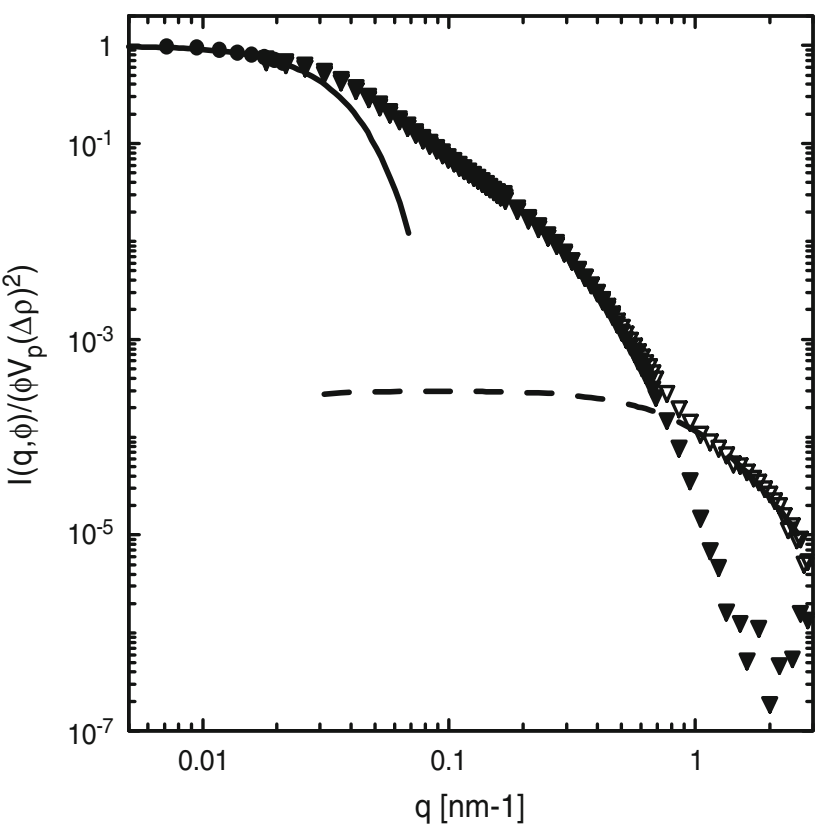

Fig. 4 Scattering intensity $I(q, \phi) /\left(\phi V_{p}(\Delta \rho)^{2}\right)$ in the limit of vanishing concentration before (open symbols) and after (filled symbols) subtraction of scattering contributions due to fluctuations (blob scattering). The circles denotes the values obtained by SLS and the inverted triangles the ones obtained from SANS. The dashed line corresponds to the blob scattering (Eq. 6). The forward scattering according to Guinier's law (Eq. 4) is given by the full line

is the scattering due to thermal fluctuations relative to the amplitude of the contribution resulting from the overall shape. $I_{\text {fluc }}(q)$ is also called blob scattering [7]. The dashed line in Fig. 4 displays the fit according to Eq. 6. The best description fitting Eq. 6 to the experimental data base on a radius of gyration of the cross section $R_{\mathrm{c}}$ of $5.0 \mathrm{~nm}$, a correlation length (blob size) $\xi$ of $1.0 \mathrm{~nm}$ and the Flory exponent $v_{b}$ of 0.6 . The value $v_{b}$ is very close to the theoretical result expected from perturbation calculations for flexible cylinders with excluded volume interactions, which is 0.588 .

The corrected intensity thus obtained can now be used to estimate the mass per unit length $M_{\mathrm{L}}$ and the cross-sectional radius of gyration $R_{\mathrm{c}}$ (see discussion of Eq. 5). Figure 5 displays the respective plot. The $q$ range in which Eq. 5 can be applied safely is rather small. However, the accuracy is sufficient to estimate $M_{\mathrm{L}}$ to $19,000 \mathrm{~g} / \mathrm{Mol} / \mathrm{nm}$. If the length of the repeating unit would be $0.25 \mathrm{~nm}$ as expected for vinylic chains, $M_{\mathrm{L}}$ follows from the degree of polymerization of 1,600 and the molecular weight of the repeating unit $M_{0}$ (see above) to $18,400 \mathrm{~g} / \mathrm{Mol} / \mathrm{nm}$. The slightly higher value of $M_{\mathrm{L}}(19,000 \mathrm{~g} / \mathrm{Mol} / \mathrm{nm})$, however, points to a length of the repeating unit of ca. $0.24 \mathrm{~nm}$. We admit that this value is affected by a significant error. Nevertheless a similar shortening of the repeating unit was found recently by Zhang and coworkers [8]. Up to now, the origins of this

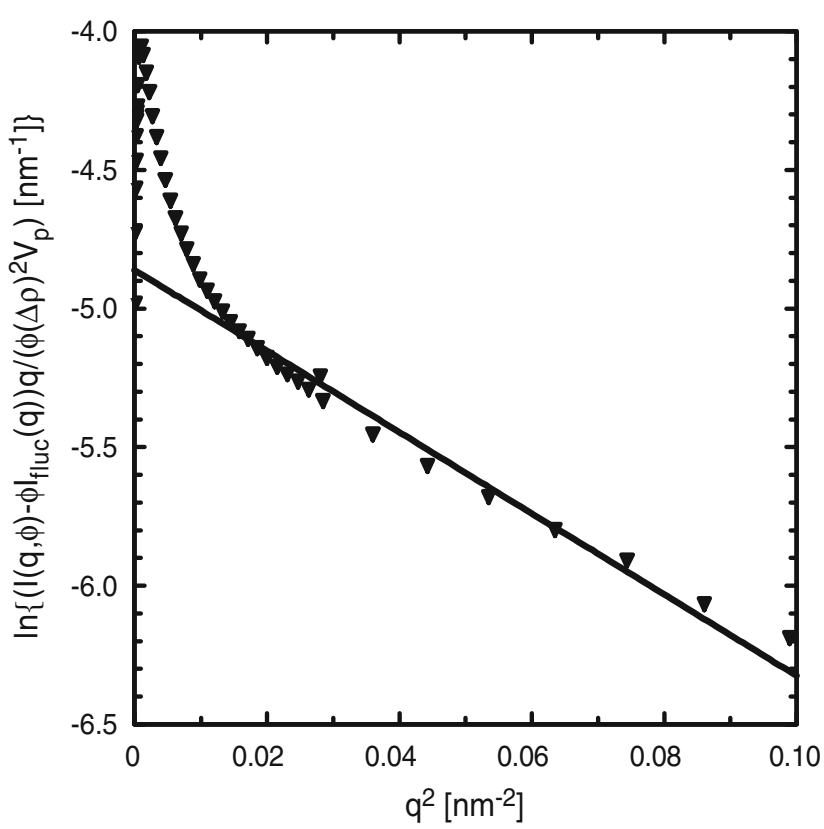

Fig. 5 Plot of the normalized scattering intensity scattering according to Eq. 5 for the determination of the mass per unit length $M_{L}$ and the cross-sectional radius of gyration $R_{c}$. The measured intensity is corrected to the blob scattering. From the linear relationship $V_{p}$ and thus $M_{L}$ and $R_{c}$ are obtained

effect are not fully clear. The cross-section radius of gyration $R_{\mathrm{c}}$ of ca. $5.4 \mathrm{~nm}$ is afflicted by a big error around $15 \%$ (cf. Fig. 5).

The investigated polymers can be described as flexible cylinders with a circular cross-section and a uniform scattering length density. Thus, for the interpretation of the scattering intensity at vanishing concentration, we used the empirical equations for semiflexible polymer chains with excluded volume interaction derived by Petersen and Schurtenberger (model 3 of reference [21]). The model is a parameterization of Monte Carlo simulations of a discrete representation of the worm-like chain model of Kratky and Porod applied to the pseudo-continuous limit. For details see Eqs. 3, 26, and 27 in the original reference [21]. The parameters of the model are the cross-sectional radius $R_{\mathrm{c}}$, the contour length $L$ (total length) and the persistence length $l_{\mathrm{p}}$. The contour length is described as a chain of some number of locally stiff segments of length $l_{\mathrm{p}}$. Hence, this value is a measure for the stiffness of the chain. Polydispersity of the cross-section is included using a Schulz-Zimm distribution. The comparison of the experimental data to the model is shown in Fig. 6.

The scattering intensity extrapolated to vanishing concentration is well-described by this model using $5.0 \mathrm{~nm}$ as radius of the cross-section as suggested by the fit of the blob scattering. The contour length results as $380 \mathrm{~nm}$ and the persistence length as $17.5 \mathrm{~nm}$. The contour length is comparable with the contour length of $384 \mathrm{~nm}$ calculated 


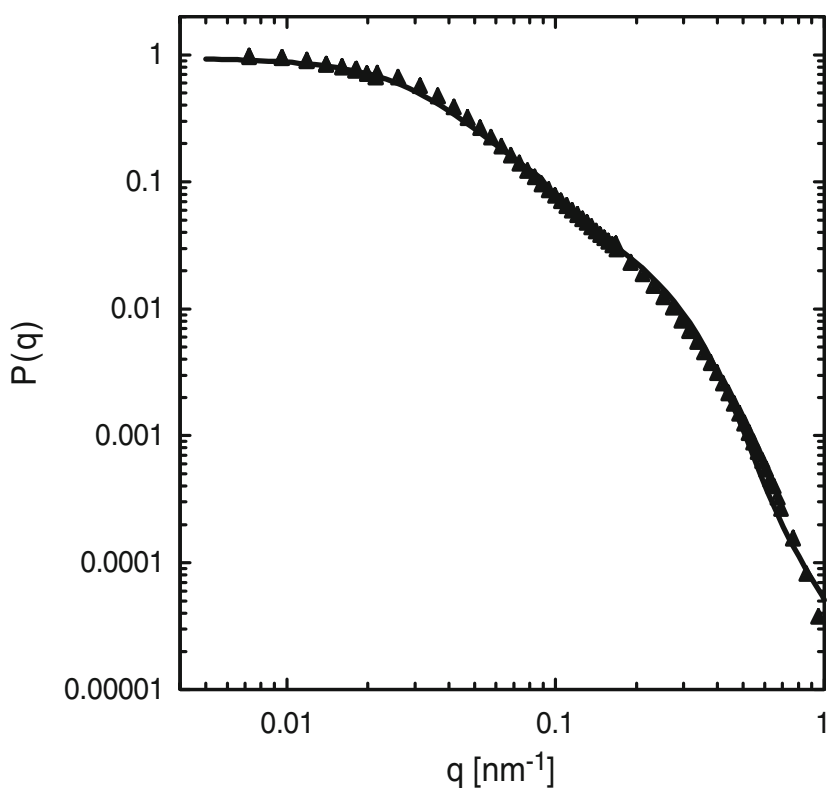

Fig. 6 Comparison of the experimental data extrapolated to zero concentration to the model of flexible cylinder with a circular cross section. The measured scattering intensity is corrected to the blob scattering. Parameters of the fit are: $L=380 \mathrm{~nm}, R_{c}=5.0 \mathrm{~nm}(\sigma=30 \%)$, and $l_{p}=17.5 \mathrm{~nm}$

from degree of polymerization and the length of the repeating unit of $0.24 \mathrm{~nm}$.

\section{Intermolecular pair correlation}

We now turn our attention to the analysis of the scattering intensities taken at finite concentration. One can calculate structural properties of interacting polymer brushes using generalized Ornstein-Zernike equations of an interaction site integral equation theory. Spatial pair correlations of a polymer brush solution are characterized by a set of intermolecular site-site total correlation functions $h_{i j}(r, \phi)$, where the indices $i$ and $j$ run over interaction sites on each of two brush polymers. These functions are related to a set of intermolecular site-site direct correlation functions $c_{i j}(r, \phi)$ by the generalized Ornstein-Zernike relations of the reference interaction site model [22]. This set of generalized Ornstein-Zernike equations must be supplemented by a set of closure relations. If the interaction sites are simply the centers of exclusion spheres, to account for steric effects, a convenient closure is the Percus-Yevick approximation [22]. The reference interaction site model has been proved to be a successful theory of the pair structure of many molecular fluids [23]. In the case of macromolecular and colloidal systems, with very large numbers of interaction sites, the number of coupled Ornstein-Zernike equations becomes intractable, and a considerable simplification follows from the assumption that the direct correlation functions $c_{i j}(r, \phi)$ are independent of the indices $i$ and $j$. This leads to the polymer reference interaction site model theory first applied by Schweizer and Curro to long flexible polymers [24]. PRISM neglects end effects in that case. The resulting single generalized Ornstein-Zernike equation of the PRISM reads

$h(q, \phi)=\frac{P^{2}(q, \phi) c(q, \phi)}{1-\frac{\phi}{V_{\mathrm{P}}} c(q, \phi) P(q, \phi)}$,

where $h(q, \phi)$ and $c(q, \phi)$ are particle-averaged total and direct correlation functions, respectively. $P(q, \phi)$ is the Fourier transform of the sum of the intramolecular twopoint correlation functions for a given volume fraction $\phi$. In the limit $\phi \rightarrow 0$ this function reduces to the form factor $P(q) \equiv P(q, \phi \rightarrow 0)$. Eq. 8 is solved numerically together with the Percus-Yevick closure taking steric interactions into account. Within the PRISM theory the structure factor $S(q, \phi)$ reads

$S(q, \phi)=1+\frac{\phi h(q, \phi)}{V_{\mathrm{p}} P(q, \phi)}$.

The scattering intensity $I(q, \phi)$ is calculated according to Eq. 1 with $S(q, \phi)$ as input. The PRISM integral equation theory has been successfully applied to various systems, such as rod-like viruses [25], plate-like colloids [26], dendrimers [16, 20], and nanoparticles [27], flexible polymers [28], mixtures of spherical colloids and semiflexible polymers [29], polyelectrolyte brushes [30]. Moreover, it has been demonstrated that the simpler PRISM theory yields results in good agreement with the more elaborate reference interaction site model calculations for lamellar colloids [31].

In Fig. 7 the experimental scattering intensity $I(q, \phi)$ is compared to the results of the integral equation theory for the PRISM. We have used the form factor $P(q)$ calculated for a worm-like chain of length $L=380 \mathrm{~nm}$ und persistence length $l_{\mathrm{p}}=17.5 \mathrm{~nm}$ as input into the generalized OrnsteinZernike equation, i.e., $P(q, \phi)=P(q)$ in Eqs. 8 and 9. For the sake of clarity the scattering intensities related to different volume fractions have been shifted vertically in Fig. 7. With increasing volume fraction the integral equation results (long dashed lines) and the experimental data (symbols) deviate. The comparison of the calculated scattering intensities with the experimental data demonstrates that the persistence length, $l_{\mathrm{p}}=17.5 \mathrm{~nm}$, and the form factor $P(q)$ may be used as input into the generalized Ornstein-Zernike equation only for very low volume fractions of the polymer brushes $\phi \leq 0.002$. For higher volume fractions marked deviations are found indicating that this approach is no longer valid.

An alternative way of modeling these data is to consider a volume fraction-dependent persistence length of polymer 


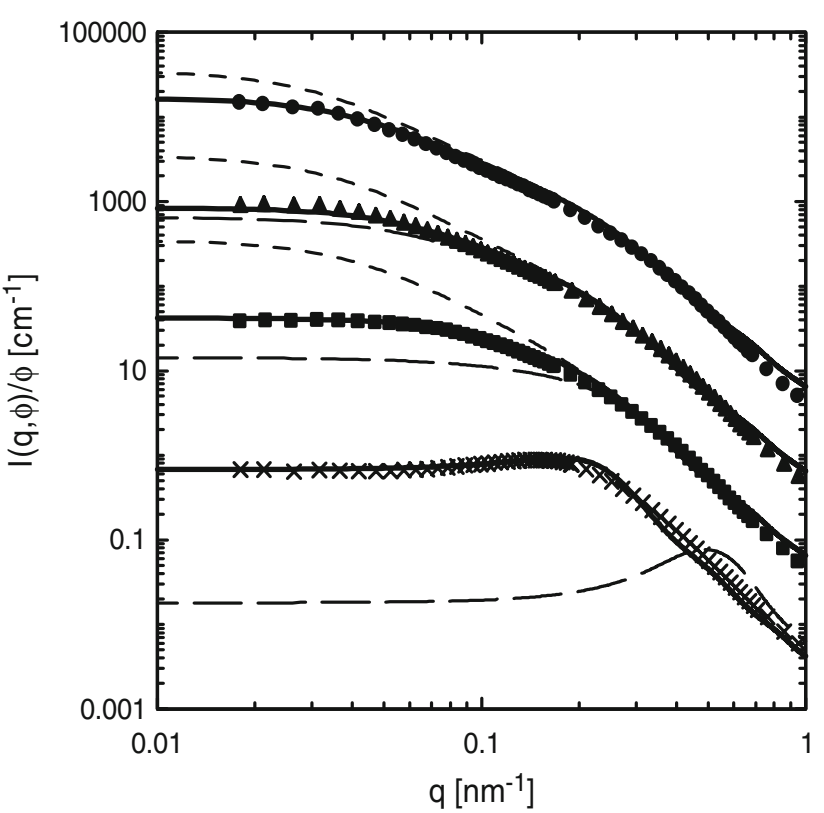

Fig. 7 Measured scattering intensity of $I(q, \phi)$ of polymer brush solutions normalized to their volume fraction $\phi$ which increases from top to bottom $(\phi=0.002,0.006,0.013,0.038)$. The three lowermost intensities are shifted down by a factor of 10, 100, and 1,000 respectively. The dashed lines represent the corresponding intensities as obtained from the PRISM integral equation theory (Eqs. 8, 9 and 1) and assuming a volume fraction-independent shape of the polymers. The solid lines represent the scattering intensities as obtained from the PRISM integral equation theory using the volume fraction-dependent persistence lengths shown in Fig. 8 with the same symbol code (solid circle, triangle, square and cross). For $\phi=0.002$ the dashed and solid curve coincide because the same persistence is used for both curves. The short dashed lines represent the scattering intensity calculated according to Eq. 1 with $S(q, \phi)=1$ for $\phi=0.002,0.006$, and 0.013

brushes and hence a volume fraction-dependent intramolecular correlation function $P(q, \phi)$ as input into Eqs. 8 and 9. Here $P(q, \phi)=P\left(q, \phi, l_{\mathrm{p}}, L\right)$ is the form factor of a semi-flexible polymer chain that depends on both the contour length $L$ and the persistence length $l_{\mathrm{P}}[21]$. The results for the scattering intensities as obtained from the PRISM integral equation theory using volume fraction-dependent persistence lengths are in agreement with the experimental data for $I(q, \phi)$ (solid lines in Fig. 7). For comparison, the short dashed lines in Fig. 7 depict the modeling of the experimental data assuming a solution of noninteracting polymer brushes characterized by $S(q, \phi)=1$ in Eq. 1 .

The dependence of the persistence length on the volume fraction shown in Fig. 8 is reminiscent of the behavior of the predicted persistence length of polyelectrolytes $[13,32]$. Although the polymer brush solutions under consideration and the theoretically investigated polyelectrolyte solutions distinctly differ from each other, there is a significant overlap between them, namely the change of the shape of the polymers upon varying the volume fraction. The

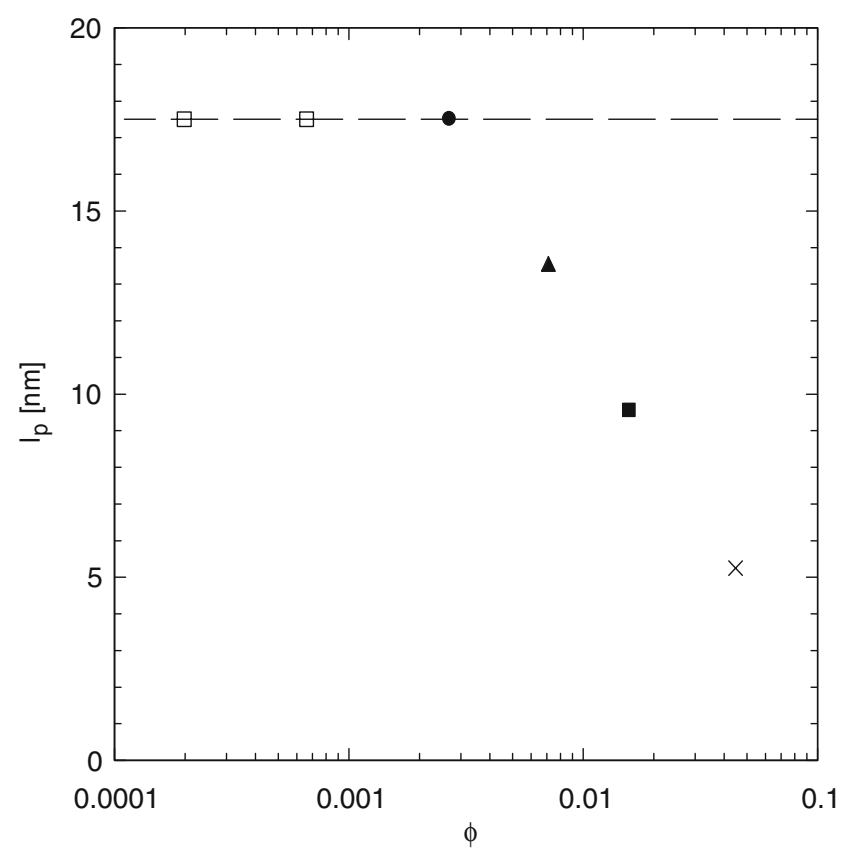

Fig. 8 The volume fraction-dependent persistence lengths used in the calculation of the scattering intensities shown by the solid lines in Fig. 7 (with the same symbol code, i.e., solid circle $l_{p}=17.5 \mathrm{~nm}$, triangle $l_{p}=13.5 \mathrm{~nm}$, square $l_{p}=9.55 \mathrm{~nm}$, and crosses $l_{p}=5 \mathrm{~nm}$ ). The open squares denote two low volume fractions which have been used for the extrapolation to infinite dilution

essential features that determine the shape of these macromolecules are the following ones: (1) The bare main chain molecules, i.e., without grafted side chains in the case of the brushes and without charges in the case of the polyelectrolyte chains, are rather flexible coils. (2) In dilute solution, the macromolecules adopt a worm-like configuration due to the steric interaction between the side chains in the case of the polymer brushes and due to the electrostatic repulsion in the case of the polyelectrolyte chains. (3) For both systems the contribution of the intermolecular interactions to the total free energy increases upon increasing the volume fraction. In order to reduce this contribution a softening of the stiffness of the macromolecules occurs because for a flexible macromolecule the excluded volume that is not available for the other macromolecules is smaller than the corresponding one of a rigid macromolecule.

Within a self-consistent integral equation theory [32-39] based on a variational method [39] the volume fractiondependent persistence length $l_{\mathrm{p}}(\phi)$ can be obtained from the self-consistent equation

$$
\begin{aligned}
& G\left[\left(l_{\mathrm{p}}(\phi), l_{\mathrm{p}}(0)\right]=\right. \\
& \quad \int_{0}^{\infty} d q q^{2} \frac{W(q, \phi)}{k_{\mathrm{B}} T} \int_{0}^{L} d s_{1} \int_{0}^{L} d s_{2}\left(s_{1}-s_{2}\right)^{2} \exp \left(-\frac{q^{2} a\left(s_{1}-s_{2}, \phi\right)}{6}\right)
\end{aligned}
$$


with

$\frac{W(q, \phi)}{k_{\mathrm{B}} T}=-\frac{\phi}{V_{\mathrm{p}}} c^{2}(q, \phi)\left(P(q, \phi)+\frac{\phi}{V_{\mathrm{p}}} h(q, \phi)\right)$

and

$a(s, \phi)=2 l_{\mathrm{P}}(\phi)|s|-2 l_{\mathrm{P}}(\phi)^{2}\left(1-\exp \left(-|s| / l_{\mathrm{P}}(\phi)\right)\right)$.

The functional $G\left[l_{\mathrm{p}}(\phi), l_{\mathrm{p}}(0)\right]$ is discussed in detail in reference [39] and a schematic presentation of the mediuminduced interaction $W(q, \phi)$ between two monomers of a polymer due concentration fluctuations of the surrounding polymers is given in Fig. 1 of reference [33]. Although the Fourier transform of the sum of the intramolecular twopoint correlation functions $P(q, \phi)$ and the particle-averaged total correlation function $h(q, \phi)$ of polymer brush solutions and polyelectrolyte solutions differ from each other, the persistence length may exhibit a similar volume fraction dependence due to the fact that the term on the right-hand side of Eq. 10 represents an integrated quantity. We have confirmed this hypothesis by performing additional numerical calculations for semi-flexible polyelectrolyte chains within the PRISM integral equation theory.

It is worthwhile to mention that in the case of rather rigid polymer brushes or polyelectrolyte chains an isotropic to nematic phase transition occurs upon increasing the volume fraction. In this case the contribution of the intermolecular interactions to the total free energy is reduced due to orientational order while the contribution of the orientational entropy to the total free energy is increased.

Finally we emphasize that our measurements corroborate the softening of the stiffness of polymer brushes due to mutual interactions predicted by Borisov et al. [12]. We have demonstrated that the volume fraction dependence of the radius of gyration $R_{\mathrm{g}}$ (see Eq. 4 ) is in agreement with scaling considerations for polymer brush solutions [14]. A similar decrease of the radius of gyration with increasing volume fraction has also been measured for flexible polystyrene chains in solution [40] and calculated for neutral polymer chains modeled as pearl necklaces of freely jointed tangent hard spheres [34, 41].

\section{Dynamic properties}

In addition to static properties we have investigated dynamic properties of the polymer brushes using dynamic light scattering and depolarized dynamic light scattering. The measured time-dependent DLS intensity autocorrelation function is a single exponential function of time for volume fractions $\phi \leq 0.002$ signaling pure translational diffusion of the polymers. No contributions of internal modes such as rotation, bending, or stretching to the dynamics have been found. Hence, one may describe the experimental data in

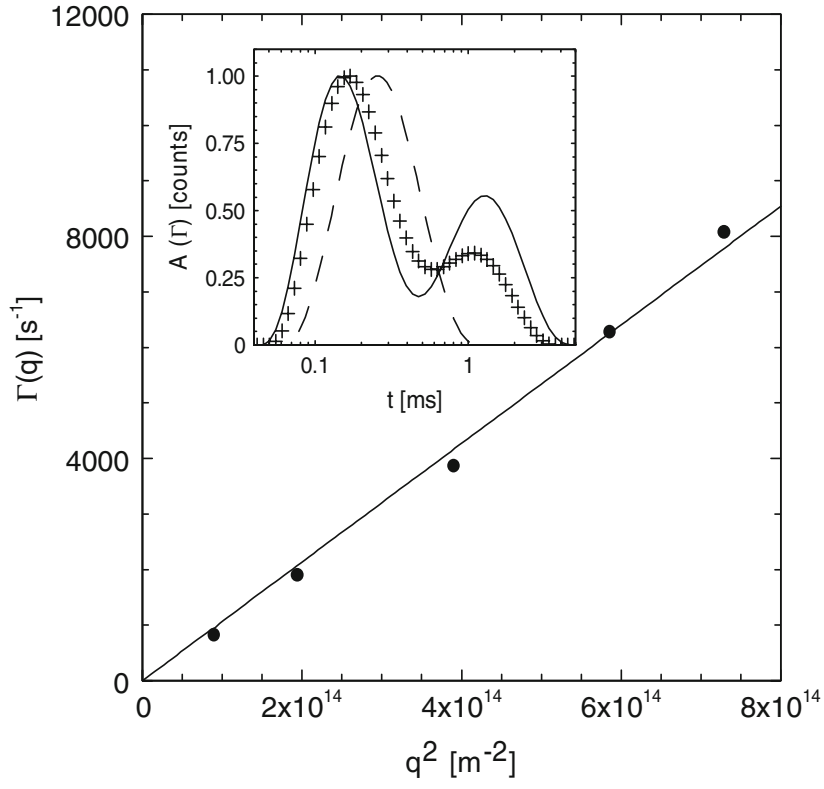

Fig. 9 Measured decay rate $\Gamma(q)=q^{2} D$ of the time-dependent intensity autocorrelation function of the polymer brush in dilute solution at the volume fraction $\phi=0.002$ as function of the square of the scattering vector $q^{2}$ (symbols). The resulting translational diffusion coefficient $D$ is given by $D=1.1 \cdot 10^{-11} \mathrm{~m}^{2} / \mathrm{s}$ (slope of the solid line). Inset CONTIN plot at $90^{\circ}$ scattering angle for $\phi=0.002$ (dashed line), 0.006 (symbols), and 0.009 (solid line)

terms of a decay rate $\Gamma(q)$ according to $\Gamma(q)=q^{2} D$, where $D$ is the translational diffusion coefficient of the polymer in dilute solution. Figure 9 shows the measured decay rate (CONTIN analysis) as a function of $q^{2}$ for the volume fraction $\phi=0.002$. The inset shows the CONTIN plots at $90^{\circ}$ scattering angle at various concentrations.

Moreover, we have determined the hydrodynamic radius $R_{\mathrm{h}}=39 \pm 2 \mathrm{~nm}$ from the measured translational diffusion coefficient using the Stokes-Einstein relation $R_{\mathrm{h}}=k_{\mathrm{B}} T /$ $(6 \pi \eta D)$, where the temperature $T$ and the viscosity $\eta$ characterize the solvent. It is instructive to compare the measured hydrodynamic radius with the results for a semiflexible chain model which has been used to interpret quasi-elastic neutron and dynamic light-scattering measurements on various natural and synthetic macromolecules such

Table 1 The experimental normalized diffusion coefficient $D^{(e x)}(\phi) / D(0)$ together with the theoretical calculations $D^{(\text {th })}(\phi) /$ $D(0)$ according to Eq. 13 and using a volume fraction-dependent shape of the polymer brush. An additional measured slow diffusive process is characterized by the normalized diffusion coefficient $D^{(\mathrm{sl})}(\phi) / D(0)$

\begin{tabular}{lccc}
\hline$\phi$ & 0.006 & 0.008 & 0.01 \\
\hline$D^{(e x)}(\phi) / D(0)$ & 1.42 & 1.53 & 1.72 \\
$D^{(\text {th })}(\phi) / D(0)$ & 1.49 & 1.64 & 1.85 \\
$D^{(\mathrm{sl})}(\phi) / D(0)$ & 0.28 & 0.24 & 0.22 \\
\hline
\end{tabular}


as denaturated ovalbumin, DNA, F-actin [42], gellan [43], polystyrene [44], xanthan [45], and worm-like micelles [46]. The numerical evaluation according to [42]

$$
\frac{1}{R_{\mathrm{h}}}=2\left(1+\frac{\sqrt{3}}{\sqrt{\pi} L} \int_{2 R}^{L} d s \frac{L-s}{a(s, \phi)} \exp \left(-\frac{3 R^{2}}{2 a(s, \phi)}\right)\right)
$$

yields $R_{\mathrm{h}}=38.5 \mathrm{~nm}$ which is comparable with the experimentally determined value. In the calculations the contour length $L=380 \mathrm{~nm}$, the persistence length $l_{\mathrm{p}}=17.5 \mathrm{~nm}$, and the radius of the cross-section $R=\sqrt{2} R_{c}=7 \mathrm{~nm}$ have been used. Moreover, we have calculated the full time-dependent intensity autocorrelation function and we have found that internal modes do not contribute for the scattering vectors used in the DLS experiments. However, internal modes do contribute for stiffer polymers confirming our findings concerning the stiffness of the polymer brushes. The DDLS intensity signal is too low to measure an intensity autocorrelation function as expected for rather flexible worm-like cylinders in contrast to rigid rods.

Finally, we study dynamic properties of the polymer brushes at higher volume fractions. The time-dependent scattering intensity may be written as

$I(q, \phi, t)=I(q, \phi, 0) \exp (-\Gamma(q, \phi) t)$

with the decay rate [47]

$\Gamma(q, \phi)=\frac{k_{\mathrm{B}} T}{4 \pi^{2} \eta} \int_{0}^{\infty} d q_{1} q_{1}^{2} \frac{I\left(q_{1}, \phi\right)}{I(q, \phi)}\left(\frac{q_{1}^{2}+q^{2}}{2 q_{1} q} \log \left|\frac{q_{1}+q}{q_{1}-q}\right|-1\right)$.

The volume fraction-dependent diffusion coefficient $D(\phi)$ can be calculated according to

$D(\phi)=\lim _{q \rightarrow 0} \frac{\Gamma(q, \phi)}{q^{2}}$.

This diffusion coefficient increases upon increasing the volume fraction of the cylindrical polymer brushes due to an increasing restoring force for concentration fluctuations as is illustrated in Table 1, where the calculated normalized diffusion coefficient $D^{(\mathrm{th})}(\phi) / D(0)$ is shown together with the corresponding measured diffusion coefficient $D^{(\mathrm{ex})}(\phi) / D(0)$ for three volume fractions.

In the calculations according to Eqs. 15 and 16 the volume fraction-dependent persistence lengths and the corresponding static scattering intensities $I(q, \phi)$ have been used (solid lines in Fig. 7). The deviations between the theoretical and experimental might be due to the fact that the hydrodynamic interaction has been taken into account in terms of the Oseen tensor in order to derive Eq. 15 [47].
Using the Rotne-Prager tensor [48] as a first correction to the Oseen tensor will improve the results. However, we emphasize that using the volume fraction-independent persistence length $l_{\mathrm{p}}=17.5 \mathrm{~nm}$ and the corresponding static scattering intensities $I(q, \phi)$ as input into Eqs. 15 and 16 does not lead to an agreement with the experimental data. The calculated diffusion coefficients are larger than both the experimental and theoretical values shown in Table 1. In addition we have observed experimentally a slow diffusive process at higher volume fractions $D^{(\mathrm{sl})}(\phi)$ in Table 1 which might be associated with long-range concentration fluctuations. The contribution of the slow mode to the timedependent intensity autocorrelation function increases upon increasing the volume fraction as it is apparent from the inset of Fig. 9.

\section{Conclusion}

We presented the full analysis of a cylindrical polymer brush in the dilute and semi-dilute regime by a combination of small-angle neutron scattering, static and dynamic light scattering. At first, the limit of infinite dilution was established by careful extrapolation to vanishing concentration. The evaluation of the SANS data taken at finite concentration then demonstrated that the persistence length is decreasing from $17.5 \mathrm{~nm}$ (infinite dilution) to $5.3 \mathrm{~nm}$ at ca. $4 \mathrm{wt} . \%$. This finding is in full agreement with the analysis of the data obtained by dynamic light scattering. The marked decrease of the persistence length with concentration is hence comparable to the decrease of this quantity of linear polyelectrolytes in the same concentration regime.

Acknowledgment Financial support by the Deutsche Forschungsgemeinschaft SFB 481, Bayreuth, is gratefully acknowledged. The authors thanks the Institute Laue-Langevin in Grenoble (France) for providing beamtime at the instrument D11.

\section{References}

1. Gerle M, Fischer K, Roos S, Müller AHE, Schmidt M, Sheiko SS, Prokhorova S, Möller M (1999) Macromolecules 32:2629

2. Wang J, Matyjaszewski K (1995) J Am Chem Soc 117:5614

3. Zhang M, Breiner T, Mori H, Müller AHE (2003) Polymer 44:1449

4. Zhang M, Drechsler M, Müller AHE (2004) Chem Mater 16:537

5. Zhang M, Müller AHE (2005) J. Polym. Sci.-Part A: Polym Chem 43:3461

6. Elli S, Ganazzoli F, Timoshenko EG, Kuznetsoy YA, Connolly R (2004) 120:6257

7. Rathgeber S, Pakula T, Wilk A, Matyjaszewski K, Beers KL (2005) J Chem Phys 122:124904

8. Zhang B, Gröhn F, Pedersen JS, Fischer K, Schmidt M (2006) Macromolecules 39:8440 
9. Wataoka I, Urakawa H, Kajiwara K, Schmidt M, Wintermantel M (1997) Polymer International 44:365

10. Rathgeber S, Pakula T, Wilk A, Matyjaszewski K, Lee HI, Beers KL (2006) Polymer 47:7318

11. Gunari N, Schmidt M, Janshoff A (2006) Macromolecules 39:2219

12. Borisov OV, Birshtein TM, Zhulina YB (1987) Polym Sci USSR 29:1552

13. Stevens MJ, Kremer K (1993) Phys Rev Lett 71:2228

14. Bolisetty S, Airaud C, Xu Y, Müller AHE, Harnau L, Rosenfeldt S, Lindner P, Ballauff M (2007) Phys Rev E 75:040803

15. Rosenfeldt S, Karpuk E, Lehmann M, Meier H, Lindner P, Harnau L, Ballauff M (2006) Chem Phys Chem 7:2097

16. Rosenfeldt S, Dingenouts N, Ballauff M, Werner N, Vögtle F, Lindner P (2002) Macromolecules 35:8098

17. Guinier A, Fournet G (1955) Small Angle Scattering of X-rays. Wiley, New York

18. Li L, Harnau L, Rosenfeldt S, Ballauff M (2005) Phys Rev E 72: 051504

19. Rosenfeldt S, Dingenouts D, Ballauff M, Lindner P, Likos CN, Werner N, Vögtle F (2002) Macromol Chem Phys 203:1995

20. Harnau L, Rosenfeldt S, Ballauff M (2007) J Chem Phys 127:014901

21. Pedersen J, Schurtenberger P (1996) Macromolecules 29:7602

22. Chandler D, Andersen HC (1972) J Chem Phys 57:1930

23. Monson PA, Morriss GP (1990) Adv Chem Phys 77:451

24. Schweizer KS, Curro JG (1987) Phys Rev Lett 58:246

25. Harnau L, Reineker P (2000) J Chem Phys 112:437

26. Harnau L, Costa D, Hansen JP (2001) Europhys Lett 53:729

27. Weber CHM, Chiche A, Krausch G, Rosenfeldt S, Ballauff M, Harnau L, Schnetmann IG, Tong O, Mecking S (2007) Nano Letters 7:2024
28. Harnau L (2001) J Chem Phys 115:1943

29. Harnau L, Hansen JP (2002) J Chem Phys 116:9051

30. Henzler K, Rosenfeldt S, Wittemann A, Harnau L, Finet S, Narayanan T, Ballauff M (2008) Phys Rev Lett 100:158301

31. Costa D, Hansen JP, Harnau L (2005) Molec Phys 103:1917

32. Shew CY, Yethiraj A (2000) J Chem Phys 113:8841

33. Schweizer KS, Honnell KG, Curro JG (1992) J Chem Phys 96:3211

34. Yethiraj A, Schweizer KS (1992) J Chem Phys. 97:1455

35. Melenkevitz J, Schweizer KS, Curro JG (1993) Macromolecules 26:6190

36. Schweizer KS, Curro JG (1997) Adv Chem Phys 98:1

37. Yethiraj A (1997) Phys Rev Lett 78:3789

38. Shew CY, Yethiraj A (1999) J Chem Phys 110:5437

39. Shew CY, Yethiraj A (1998) J Chem Phys 108:1184

40. Daoud M, Cotton JP, Farnoux B, Jannink G, Sarma G, Benoit H, Duplessix R, Picot C, de Gennes PG (1975) Macromolecules 8:804

41. Yethiraj A, Hall CK (1992) J Chem Phys 96:797

42. Harnau L, Winkler RG, Reineker P (1996) J Chem Phys 104:6355

43. Harnau L, Winkler RG, Reineker P (1997) Macromolecules 30:6974

44. Harnau L, Winkler RG, Reineker P (1998) J Chem Phys 109:5160

45. Harnau L, Winkler RG, Reineker P (1999) Macromolecules 32:5956

46. Berlepsch H, Harnau L, Reineker P (1998) J Phys Chem B 102:7518

47. Doi M, Edwards SF (1986) The Theory of Polymer Dynamics. Clarendon, Oxford

48. Rotne J, Prager S (1969) J Chem Phys 50:4831 